\title{
Application of Dual-Energy CT Non-Linear Fusion Technology in Improving CTA Image Quality of Renal Cancer
}

\author{
Shuiqing Zhuo' ${ }^{\text {, Xiaoling Chen }}{ }^{2}$, Jingping Yu${ }^{1}$, Sihui Zeng ${ }^{1}$, Lizhi Liu ${ }^{1}$ \\ ${ }^{1}$ State Key Laboratory of Oncology in South China, Collaborative Innovation Center for Cancer Medicine, Department of Imaging \\ Sciences of Sun Yat-sen University Cancer Center, Guangzhou, China \\ ${ }^{2}$ Joint Surgery, The First Affiliated Hospital of Sun Yat-sen University, Guangzhou, China \\ Email: zhuoshq@sysucc.org.cn
}

How to cite this paper: Zhuo, S.Q., Chen, X.L., Yu, J.P., Zeng, S.H. and Liu, L.Z. (2018) Application of Dual-Energy CT Non-Linear Fusion Technology in Improving CTA Image Quality of Renal Cancer. Open Journal of Medical Imaging, 8, 73-80.

https://doi.org/10.4236/ojmi.2018.83009

Received: September 4, 2018

Accepted: September 22, 2018

Published: September 25, 2018

Copyright $\odot 2018$ by authors and Scientific Research Publishing Inc. This work is licensed under the Creative Commons Attribution International License (CC BY 4.0).

http://creativecommons.org/licenses/by/4.0/

\begin{abstract}
Objective: To explore the significance of dual-energy CT non-linear fusion technique in improving the quality of CTA image of renal cancer. Methods: The CTA images of 100 patients who had been confirmed by pathology as renal cancer were collected and were randomly divided into experimental group and control group with 50 cases respectively. The two groups of patients were treated with iodine concentration of $300 \mathrm{mg} / \mathrm{ml}$ and $350 \mathrm{mg} / \mathrm{ml}$ non-ionic contrast agent, with a dosage of $1.5 \mathrm{ml} / \mathrm{kg}$ and an injection rate of 4 $\mathrm{ml} / \mathrm{s}$. The contrast agent intelligently tracking method was adopted bolus. The control group used the conventional CTA scanning, with a reference tube voltage/tube current of $100 \mathrm{kv} / \mathrm{ref} 150$ mas. The experimental group adopted the double energy scanning, with ball tube $\mathrm{A}$ and ball tube $\mathrm{B}$. The reference tube voltage/tube current was $100 \mathrm{kv} / \mathrm{ref} 250$ mas and sn150 $\mathrm{kv} / \mathrm{ref} 125$ mas respectively. The images of the experimental group were non-linear fused to obtain the Mono+ 55 kev single-energy images. The CT value, SNR contrast ratio of the abdominal aorta, renal artery and tumor tissue of the experimental group images and the $100 \mathrm{KV}$ images and the Mono+ $55 \mathrm{kev}$ images of the control group were compared. The objective evaluation and subjective evaluation of the image quality of the three groups of images was performed. Results: The results showed that the $100 \mathrm{kV}$ images of the experimental group were statistically different from those of the control group (P05) in CT value, SNR and CNR $(\mathrm{P}<0.05)$. And there was no statistically significant difference between the non-linear fusion single-energy Mono+ $55 \mathrm{kev}$ images and the control group images in CT value, SNR and CNR $(P>0.05)$. The subjective evaluation of image quality showed that there was no significant difference between Mono+ $55 \mathrm{kev}$ images and control
\end{abstract}


group images, and the quality of Mono+ $55 \mathrm{kev}$ images was higher than that of experimental group $100 \mathrm{kV}$ images. Conclusion: The dual-energy CT non-linear fusion technique can improve the quality of CTA image in patients with renal cancer, and it is possible to obtain high quality CTA images with low iodine concentration contrast agent.

\section{Keywords}

Dual-Source CT, Non-Linear Fusion Technology, Renal Cancer, Computed Tomographic Angiography, Image Quality

\section{Introduction}

With the wide application of dual-energy CT and the continuous innovation of technology, different post-processing and analysis algorithms have been continuously developed. The generation of virtual single-energy images by non-linear fusion technology is one of the important post-processing applications of dual-energy CT [1]. Due to the strong absorption ability of high atomic number substances, such as iodine contrast agent to low-energy X-ray photons, in low-energy single energy spectrum images, contrast agent enhanced blood vessels and lesions and other tissues can be used to optimize the display of lesions. In recent years, this new technique has been reported continuously in abdominal clinical applications [2] [3] [4]. It is believed that this technique can improve image contrast and blood vessel display quality, reduce noise and improve image quality as well as the detection rate of small lesions. Thus it is a promising technique. The purpose of this paper is to study the significance of dual-energy CT non-linear fusion technology in improving CTA image quality of renal cancer patients, and to achieve high-quality CTA image with low-concentration iodine contrast agent.

\section{Materials and Methods}

\subsection{General Data}

CTA images of 100 patients with renal cancer confirmed by pathology were collected, including 65 males and 45 females, aged from 32 to 89 years, with an average age of $57.6 \pm 15.87$ years. Among them, 50 cases took iodine concentration of $350 \mathrm{mg} / \mathrm{ml}$ and the other 50 cases took $300 \mathrm{mg} / \mathrm{ml}$. These cases were classified into control group and experimental group.

\subsection{Scanning Modes}

All patients underwent kidney CTA scanning with Siemens Dual-source CT. Among it, the control group underwent conventional CTA scanning with a reference tube voltage/tube current of $100 \mathrm{kV} /$ ref 150 mas, a bulb rotation speed of $0.5 \mathrm{~s}$ and a pitch of 1 ; collimation width: $192 \times 0.6 \mathrm{~mm}$; reconstruction thickness: 
$1 \mathrm{~mm}$. The experimental group adopted dual-energy scanning, with bulb tube A and bulb tube $\mathrm{B}$. The reference voltage/tube current of the two bulb tube were $100 \mathrm{kV} /$ ref250 mAs and Sn150 kV/ref125 mAs, respectively. Ball tube rotation speed was $0.5 \mathrm{~s}$; pitch was 1 ; collimation width: $2 \times 192 \times 0.6 \mathrm{~mm}$; reconstruction thickness: $1 \mathrm{~mm}$. Group A images and group B images generated automatically. The patient took the supine position with foot-first mode. The American MEDRAD Stellant CT special high-pressure syringe was used with a contrast agent dose of $1.5 \mathrm{ml} / \mathrm{kg}$ body weight and a bolus injection rate of $4 \mathrm{ml} / \mathrm{s}$. Before bolus injection of contrast agent, $20 \mathrm{ml}$ of normal saline was injected at the same speed to test the patency of blood vessels, and $20 \mathrm{ml}$ of normal saline was injected after injection of contrast agent. Contrast agent bolus intelligent tracking technology was adopted. The tracking point was set at the abdominal descending aorta $2 \mathrm{~mm}$ below the diaphragm, with a ROI area of about $1 \mathrm{~mm} \cdot \mathrm{m}^{2}$ and a trigger threshold of $100 \mathrm{Hu}$. The trigger scan started 7 seconds after the enhancement of the region of interest reached the threshold. The scanning range was 20 $\mathrm{mm}$ below the diaphragm to the level of the anterior superior iliac spine.

\subsection{Image Quality Evaluation}

The images of experimental group A and B were fused nonlinearly to obtain Mono+ $55 \mathrm{keV}$ single energy images. The images of control group, $100 \mathrm{kV}$ images and Mono+ $55 \mathrm{keV}$ images of the experimental group were compared and analyzed for three-dimensional reconstruction to obtain VRT and MIP images.

Subjective evaluation: two senior image diagnosticians used the double-blind method to evaluate the cross-sectional images, VRT and MIP images of the above three groups of images at the workstation using Syngo.via. Classification criteria for CT display of renal artery branches [5]: the main renal artery is an tier-one branch; the anterior and posterior branches of the renal artery are the tier-two branches; the segmental artery is the tier-three branch; the fine branches below the segment branches are tier-four to tier-five branches. In this study, the image quality score of renal artery was divided into four grades according to the above grades: display of tier-one branch was one point; display of tier-two branch was two points; display of tier-three branches was three points; display of tier-four to tier-five branches was 4 points. And 5-point evaluation was adopted for Tumor contour and Image quality: 1 point: the image quality is very poor and cannot be used for evaluation at all; 2 points: the image quality is poor, and can basically be used for evaluation; 3 points: the image quality is general and can be tolerated, but is affected by appropriate amount of noise, artifacts and image distortion; 4 points: the image quality is good. The noise, artifact and image distortion does not affect the clinical diagnosis; 5 points: the image quality is very good. There is low noise, good contrast, no artifact and no image distortion, which completely meets the clinical diagnostic requirements.

Objective evaluation: Syngo. VIA software was adopted. The circular region of interest (ROI) in the abdominal aorta, renal artery, subcutaneous fat and renal 
tumor tissues at the level of renal hilum respectively, and was selected to measure CT values. The CT values of the same part were measured repeatedly for 3 times to obtain the average value, and the ROI was placed in the center of the blood vessel with the diameter not exceeding $2 / 3$ of the diameter of the blood vessel to be measured; standard deviation (SD) of fat CT value was set as image noise; tumor contrast noise ratio (CNR) was (tumor CT value - fat CT value)/SD; SNR was CT blood vessel/SD. Based on that, CNR and SNR of abdominal aorta and renal artery were calculated.

\subsection{Statistical Analysis}

SPSS 20 software was used for statistical processing. The measurement data of each group was expressed as mean standard deviation. The differences of SNR, $\mathrm{CNR}, \mathrm{SD}$ and subjective scores of the images of the three scanning modes were compared by Kruskal-Wallis test, and $\mathrm{P} \leq 0.05$ indicated statistically significant difference. The consistency of the two image scores was analyzed by weighted Kappa. Kappa > 0.75 indicated excellent consistency; $0.40 \leq$ kappa $\leq 0.75$ indicated good consistency and kappa $<0.40$ indicated poor consistency.

\section{Results}

$100 \mathrm{kV}$ original images of experimental group were compared with the objective image indexes of the control group. The results showed significant statistical difference $(P<0.05)$ (Table 1$)$. There was no statistically significant difference in CT value, SNR, CNR, SD of the Mono+ $55 \mathrm{kev}$ images of the experimental group and the control group images $(P>0.05)$ (Table 2). Similarly, there was statistical difference between the $100 \mathrm{kV}$ original images of experimental group and the control group images in subjective score $(P>0.05)$; And there was no statistically significant difference between the Mono+ $55 \mathrm{kev}$ images of the experimental group and the control group images in subjective score $(\mathrm{P}>0.05)$ (Table 3).

Table 1. Comparison of CT Value, SNR and CNR between $100 \mathrm{kV}$ Original Images of Experimental Group and Control Group Images and Analysis $(\bar{x} \pm s)$.

\begin{tabular}{cccc}
\hline & $\begin{array}{c}\text { Experimental group } \\
100 \mathrm{kV}\end{array}$ & $\begin{array}{c}\text { Control group } \\
100 \mathrm{kV}\end{array}$ & P-value \\
\hline Abdominal Aorta CT-Value & $407.85 \pm 74.59$ & $596.29 \pm 90.78$ & $\mathrm{P}>0.05$ \\
Abdominal Aorta SNR & $13.46 \pm 3.47$ & $18.79 \pm 5.05$ & $\mathrm{P}>0.05$ \\
Abdominal Aorta CNR & $34.71 \pm 7.36$ & $45.40 \pm 6.9$ & $\mathrm{P}>0.05$ \\
Renal Artery CT-Value & $380.31 \pm 76.81$ & $566.18 \pm 189.21$ & $\mathrm{P}>0.05$ \\
Renal Artery SNR & $10.39 \pm 4.75$ & $16.65 \pm 6.96$ & $\mathrm{P}>0.05$ \\
Renal Artery CNR & $32.87 \pm 7.28$ & $45.77 \pm 7.08$ & $\mathrm{P}>0.05$ \\
Renal Tumor CT-Value & $142.6 \pm 70.19$ & $245.89 \pm 84.14$ & $\mathrm{P}>0.05$ \\
Renal Tumor SNR & $3.63 \pm 2.31$ & $5.78 \pm 4.61$ & $\mathrm{P}>0.05$ \\
Renal Tumor CNR & $16.68 \pm 4.35$ & $20.68 \pm 6.75$ & $\mathrm{P}>0.05$ \\
\hline
\end{tabular}


Table 2. Comparison of CT Value, SNR and CNR between Mono+ $55 \mathrm{keV}$ Images of Experimental Group and Control Group Images and Analysis $(\bar{x} \pm s)$.

\begin{tabular}{cccc}
\hline & $\begin{array}{c}\text { Experimental group } \\
\text { Mono }+55 \mathrm{kV}\end{array}$ & $\begin{array}{c}\text { Control group } \\
100 \mathrm{kV}\end{array}$ & P-value \\
\hline Abdominal Aorta CT-Value & $602.34 \pm 89.67$ & $596.29 \pm 90.78$ & $\mathrm{P}<0.05$ \\
Abdominal Aorta SNR & $18.75 \pm 6.02$ & $18.79 \pm 5.05$ & $\mathrm{P}<0.05$ \\
Abdominal Aorta CNR & $44.50 \pm 7.01$ & $45.40 \pm 6.9$ & $\mathrm{P}<0.05$ \\
Renal Artery CT-Value & $568 \pm 176.39$ & $566.18 \pm 189.21$ & $\mathrm{P}<0.05$ \\
Renal Artery SNR & $16.68 \pm 6.87$ & $16.65 \pm 6.96$ & $\mathrm{P}<0.05$ \\
Renal Artery CNR & $43.78 \pm 7.43$ & $45.77 \pm 7.08$ & $\mathrm{P}<0.05$ \\
Renal Tumor CT-Value & $259.12 \pm 78.68$ & $245.89 \pm 84.14$ & $\mathrm{P}<0.05$ \\
Renal Tumor SNR & $5.80 \pm 4.78$ & $5.78 \pm 4.61$ & $\mathrm{P}<0.05$ \\
Renal Tumor CNR & $21.15 \pm 7.11$ & $20.68 \pm 6.75$ & $\mathrm{P}<0.05$ \\
\hline
\end{tabular}

Table 3. Comparison and Analysis of Subjective Image Quality Score between $100 \mathrm{kV}$ Original Images of Experimental Group and Control Group Images $(\bar{x} \pm s)$.

\begin{tabular}{cccc}
\hline & $\begin{array}{c}\text { Control group } \\
100 \mathrm{kV}\end{array}$ & $\begin{array}{c}\text { Experimental group } \\
100 \mathrm{kV}\end{array}$ & $\begin{array}{c}\text { Experimental group } \\
\text { Mono }+55 \mathrm{kV}\end{array}$ \\
\hline Renal Artery display & $4.47 \pm 0.56$ & $3.98 \pm 0.56$ & $4.36 \pm 0.56$ \\
Tumor contour & $3.97 \pm 0.54$ & $3.40 \pm 0.78$ & $4.05 \pm 0.37$ \\
Image quality & $4.18 \pm 0.42$ & $3.67 \pm 0.68$ & $4.16 \pm 0.63$ \\
\hline
\end{tabular}

\section{Discussions}

Renal cancer, also known as renal cell cancer, is the most common malignant tumor of renal parenchyma, accounting for about $3.5 \%$ of the malignant tumors of the whole body [6]. The treatment of renal cancer is mainly surgical resection or laparoscopic radical nephrectomy [7]. Before operation, clarification of the existence and shape of renal artery variation, the relationship between tumor and blood vessel, the presence or absence of pathological changes in renal blood vessel is of great significance to the selection of operation methods and the reduction of complications [8]. CTA has been considered as a common method for preoperative evaluation of radical nephrectomy. In order to obtain high-quality blood vessel images, traditional CTA usually uses high-concentration iodine contrast agents, such as iodine concentration of $350 \mathrm{mg} / \mathrm{ml}, 370 \mathrm{mg} / \mathrm{ml}$ or more. Most of the iodine contrast agents are excreted through the kidney. A large amount of high-concentration iodine contrast agents will undoubtedly increase the burden on the kidney and bring about the risk of renal damage to renal cancer patients. The purpose of this paper is to study the effect of dual-energy CT non-linear fusion technology in improving CTA image quality of renal cancer patients, so as to achieve the purpose of obtaining high quality with low iodine concentration contrast agent.

The third-generation dual-source CT non-linear fusion technology can transform two-energy image into single energy at any energy level within $40 \mathrm{kev}-190$ kev (virtual monoenergetic reconstructions Mono+) image. The lower the ener- 
gy level is, the stronger the image contrast is. Thus, the contrast-enhanced blood vessels and lesions have a better contrast than the normal single-energy CT scan in the low-power single-spectrum image. In recent years, the application of this technology has been reported constantly. Through the study of a group of pancreatic tumor patients, Davide Bellini and other scholars found that when the energy value was less than $70 \mathrm{keV}$, Mono+ could overcome the significant noise of normal Mono images to improve image quality. The objective image quality evaluation showed that the best contrast-noise ratio could be obtained in the Mono+ $40 \mathrm{kev}$ energy level. The subjective image quality evaluation considered that the optimal image quality could be obtained in Mono+ $55 \mathrm{kev}$ energy level [9]. Similarly, the study of Claudia Frellese and other scholars on the image quality between pancreatic cancer lesions and normal pancreatic tissue also found that the optimal image quality was obtained in the Mono+ $55 \mathrm{kev}$ energy value [4]. And the application of renal tumor CTA is relatively rare.

In this paper, the quality of CTA image of renal cancer patients taking low iodine concentration $(300 \mathrm{mg} / \mathrm{ml})$ contrast agent and high iodine concentration $(350 \mathrm{mg} / \mathrm{ml})$ contrast agent respectively was compared by retrospective study. It was found that the image quality of patients taking high iodine concentration contrast agent was better. There was statistically significant difference in CT value, SNR, CNR and other objective image quality evaluation indexes between the two groups. There was also statistically significant difference in subjective image quality score between the two groups. Images quality of patients taking low iodine concentration contrast agent was evaluated after the non-linear fusion to generate virtual single energy images (Mono+ $55 \mathrm{kev}$ ). There was no significant difference in CT value, SNR, CNR and other objective image quality evaluation indexes compared with those of the control group, and there was no significant difference in subjective image quality scores between the two groups of images (Figure 1).

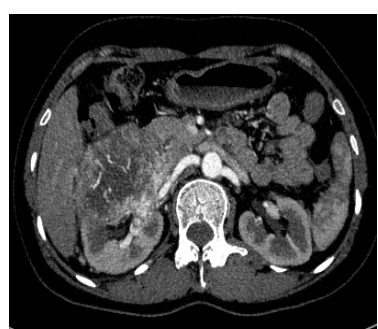

(a)

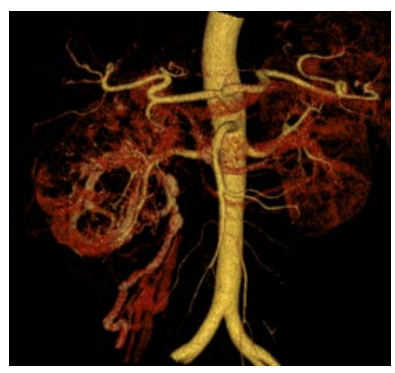

(d)

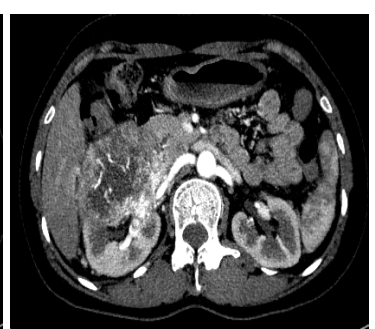

(b)

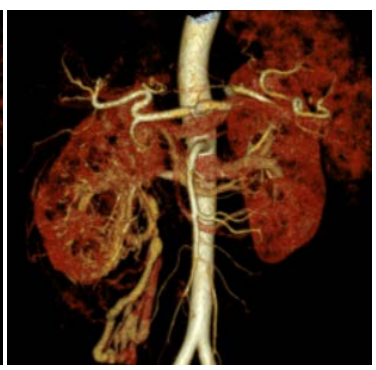

(e)

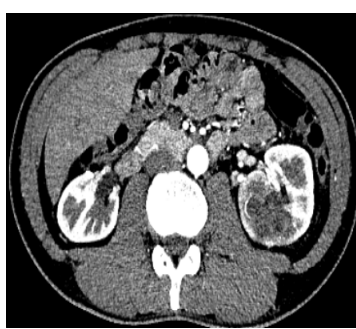

(c)

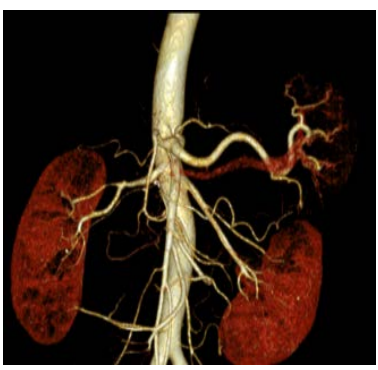

(f) 


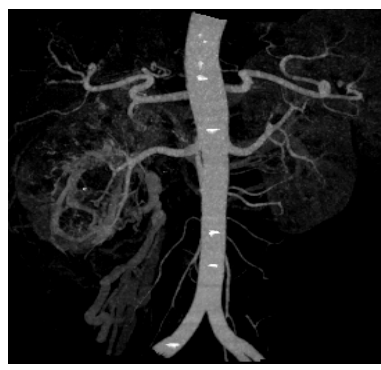

(g)

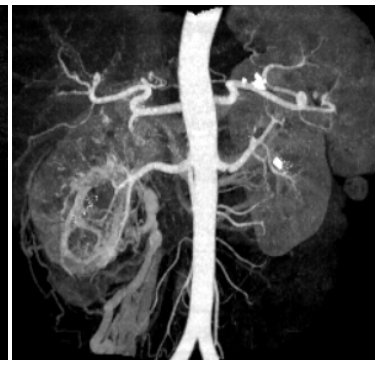

(h)

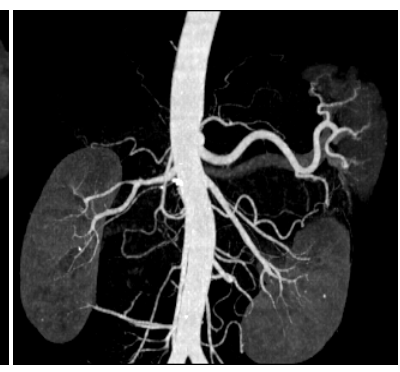

(i)

Figure 1. (a) to (i) were transverse section, VRT and MIP images respectively of Experimental group $100 \mathrm{kV}$, Experimental group Mono+ $55 \mathrm{KeV}$ and Control group $100 \mathrm{kV}$ respectively. It was shown that Experimental group $100 \mathrm{kV}$ images were significantly higher than that of Control group $100 \mathrm{kV}$ images, but the images of Experimental group Mono+ $55 \mathrm{KeV}$ was not found to be different with the images of Control group $100 \mathrm{kV}$.

To sum up, the dual-energy CT non-linear fusion technique can improve the CTA image quality of patients with renal cancer and increase the contrast between blood vessel and surrounding tissue, tumor and surrounding tissue, so that patients with low iodine concentration contrast agent can have CTA images with the same quality as that of patients with high iodine concentration. It is undoubtedly possible to reduce the risk of hepatic and renal impairment in patients with low iodine concentration contrast agent. Therefore, the dual-energy CT non-linear fusion technology is worthy of popularization.

\section{Conflicts of Interest}

The authors declare no conflicts of interest regarding the publication of this paper.

\section{References}

[1] Lv, G.M., Zhang, L.J., et al. (2016) Guidelines for Clinical Application of Dual-Energy CT. People's Health Press, No. 1, 6-8.

[2] Schabel, C., Bongers, M., Sedlmair, M., et al. (2014) Assessment of the Hepatic Veins in Poor Contrast Conditions Using Dual Energy CT: Evaluation of a Novel Monoenergetic Extrapolation Software Algorithm. Fortschr Röntgenstr, 186, 591-597.

[3] Andrew, D., Hardie, M.D., Melissa, M., et al. (2015) Application of an Advanced Image-Based Virtual Monoenergetic Reconstruction of Dual Source Dual-Energy CT Data at Low keV Increases Image Quality for Routine Pancreas Imaging. Journal of Computer Assisted Tomography, 39, 716-720.

https://doi.org/10.1097/RCT.0000000000000276

[4] Frellesena, C., Fesslera, F., Andrew, D., et al. (2015) Dual-Energy CT of the Pancreas: Improved Carcinoma-to-Pancreas Contrast with a Noise-Optimized Monoenergetic Reconstruction Algorithm. European Journal of Radiology, 84, 2052-2058. https://doi.org/10.1016/j.ejrad.2015.07.020

[5] Liu, B., Zhao, H., Wu, X.W., et al. (2006) Application of 64-Slice Spiral CT in Renal Artery Angiography. Journal of Anhui Medical University, 41, 573-575.

[6] Jemal, A., Siegel, R., Ward, E., et al. (2014) Caner Statistics, 2008. CA: A Cancer 
Journal for Clinicians, 64, 9-29.

[7] Liu, D.J., Shao, S.X. and Niu, H.T. (2012) Analysis of Surgical Complications of Retroperitoneal Laparoscopic Radical Nephrectomy and Partial Nephrectomy. Journal of Hebei Union University (Medical Edition), 14, 232-233.

[8] Chen, C., Guo, H.R., Xu, B., et al. (2017) Application of Volume CTA before Renal Tumor Surgery and Its Comparison with Spiral CTA. China Medicine and Pharmacy, 1, 16-19.

[9] Bellini, D., Gupta, S., Ramirez-Giraldo, J.C., Marin, D., et al. (2017) Use of a Noise Optimized Monoenergetic Algorithm for Patient-Size Independent Selection of an Optimal Energy Level during Dual-Energy CT of the Pancreas. Journal of Computer Assisted Tomography, 41, 39-47. 